\title{
Emerging Technologies, Infrastructures, and Applications for Video Streaming over Future Communication Networks
}

\author{
Yuanlong Cao $\mathbb{D}^{1},{ }^{1}$ Yuming Fang, ${ }^{2}$ Jiyan $W u,{ }^{3}$ Wei Quan, ${ }^{4}$ and Kai Wang ${ }^{5}$ \\ ${ }^{1}$ Jiangxi Normal University, Nanchang, China \\ ${ }^{2}$ Jiangxi University of Finance and Economics, China \\ ${ }^{3}$ Singapore University of Technology and Design, Singapore \\ ${ }^{4}$ University of Waterloo, Waterloo, Canada \\ ${ }^{5}$ Tsinghua University, China
}

Correspondence should be addressed to Yuanlong Cao; ylcao@jxnu.edu.cn

Received 17 December 2018; Accepted 17 December 2018; Published 25 February 2019

Copyright (C) 2019 Yuanlong Cao et al. This is an open access article distributed under the Creative Commons Attribution License, which permits unrestricted use, distribution, and reproduction in any medium, provided the original work is properly cited.

The objective of this special issue is to provide a premier forum for researchers working on emerging technologies and applications for video streaming to present their recent research results. A substantial number of articles were submitted, and after a thorough peer review process, eight highquality articles were selected to be included in this special issue. These papers cover emerging technologies and applications for content-rich real-time video streaming services, including QoS prediction mechanism, multimedia content transmission technologies, multimedia object detection, and so on. We believe that the research articles collected in this special issue will introduce readers to the recent advancements and interesting research directions in the field of multimedia technologies.

The paper by G. X. Hu et al. introduces a new small image objection detection model to achieve good image detection accuracy. The proposed model not only ensures the integrity of the feature of the large object but also preserves the full detail feature of the small objects by extracting the multiscale feature of the image.

The paper by R. Liu et al. presents a novel homomorphic encryption technology-based method for verification of video data integrity. The simulation results show that the proposed method is superior to comparison schemes in all aspects, and it suggests that the proposed scheme is serving better for the video data integrity verification purpose in the cloud environment.
The paper by T. Yi and C. Fang jointly combines the Matter Element Analysis (MEA) method, fuzzy set theory, and TOPSIS (Technique for Order Preference by Similarity to Ideal Solution) to design a fuzzy matter element model, which can be used to evaluate the complexity of UML class diagram and predict the complexity of software quality.

The paper by $\mathrm{W}$. Lu et al. proposes a priority-aware multipath TCP (MPTCP)-based data distribution solution in order to provide multimedia applications with many attractive benefits including goodput performance improvement, latency reduction, and high-quality users' quality of experience for multimedia streaming services.

The paper by Y. Song et al. introduces an improved artificial bee colony (ABC) algorithm for efficient multimedia streaming over wireless network environments. The experimental results show that the proposed algorithm not only ensures the real-time transmission requirement, but also reduces the power consumption of wireless network.

The paper by Q. Liu et al. presents a novel loss-aware multipath data delivery solution, which extends to the Stream Control Transmission Protocol (SCTP)'s concurrent multipath transfer mechanism, necessitating the following aims: (i) providing SCTP-CMT with a loss-aware bandwidth aggregation and parallel transmission mechanism and (ii) improving data delivery performance in a heterogeneous wireless network environment. 
The paper by W. Ma et al. first introduces a novel neural collaborative filtering model for QoS prediction using transfer learning technology. Next, the authors design a novel interaction layer to represent the relationship between latent embedding factors of the nodes. Finally, the authors utilize partial fine-tuning and maximum mean discrepancy (MMD) measurement to train the target domain model to implement domain adapting. The experimental results show that the proposed model can outperform the existing QoS prediction models in a video streaming P2P network environment.

The paper by W. Wang et al. regards a video streaming scheduling event in wireless networks as a soft real-time scheduling event and then proposes a dynamic soft real-time scheduling mechanism combined with preemption threshold for streaming media. The simulation results demonstrate the benefits of applying the proposed mechanism to a soft realtime system.

\title{
Conflicts of Interest
}

The authors declare that there are no conflicts of interest regarding the publication of this paper.

\section{Acknowledgments}

We would like to thank all the authors who contributed to this special issue. This publication would not be possible without the participation of our expert reviewers, who provided valuable feedback and constructive comments throughout the review process.

\author{
Yuanlong Cao \\ Yuming Fang \\ Jiyan $W u$ \\ Wei Quan \\ Kai Wang
}




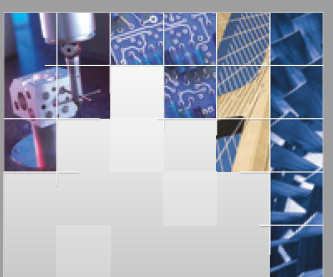

\section{Enfincering}
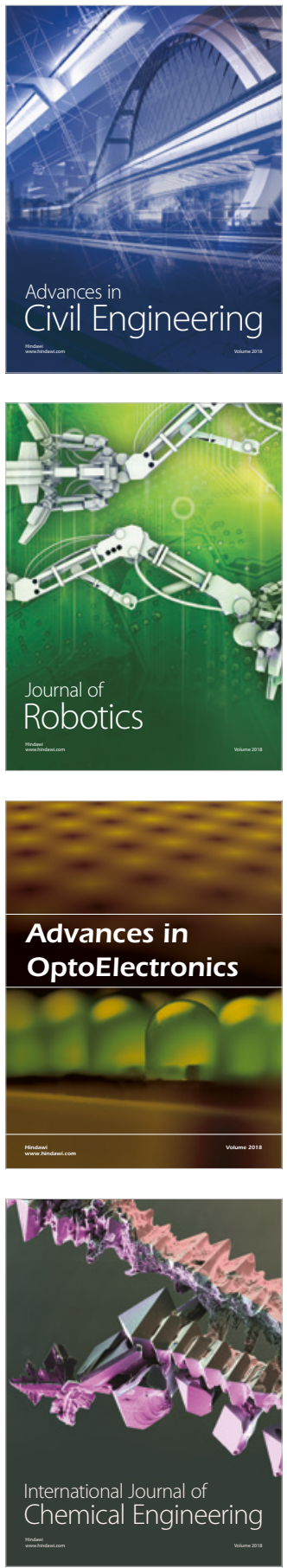

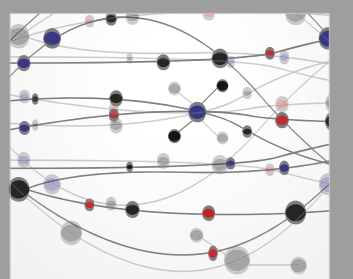

\section{Rotating \\ Machinery}

The Scientific World Journal

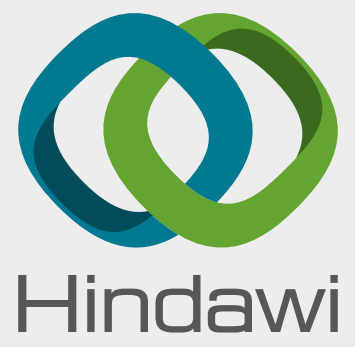

Submit your manuscripts at

www.hindawi.com
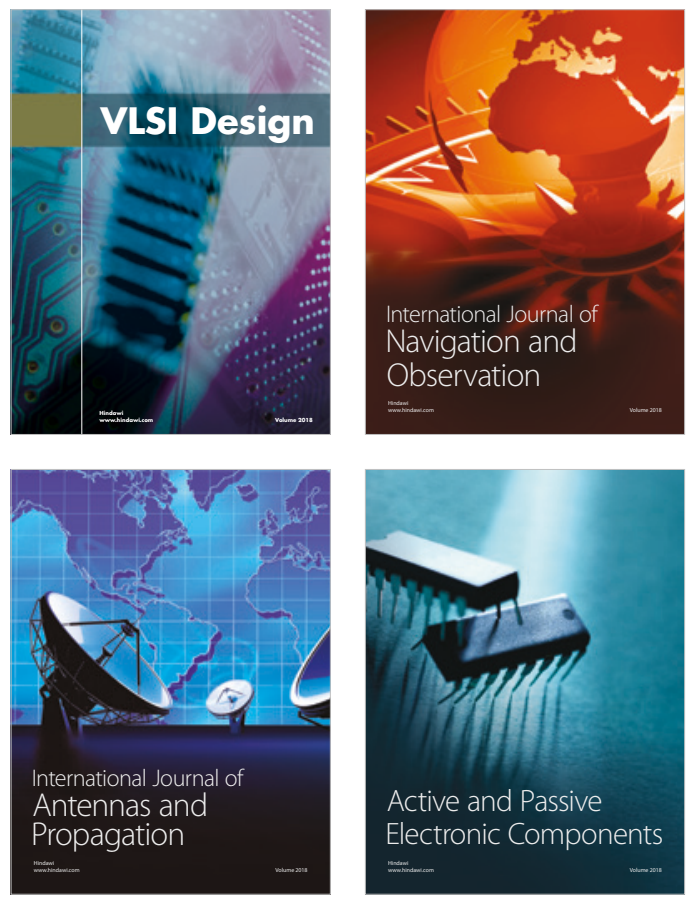
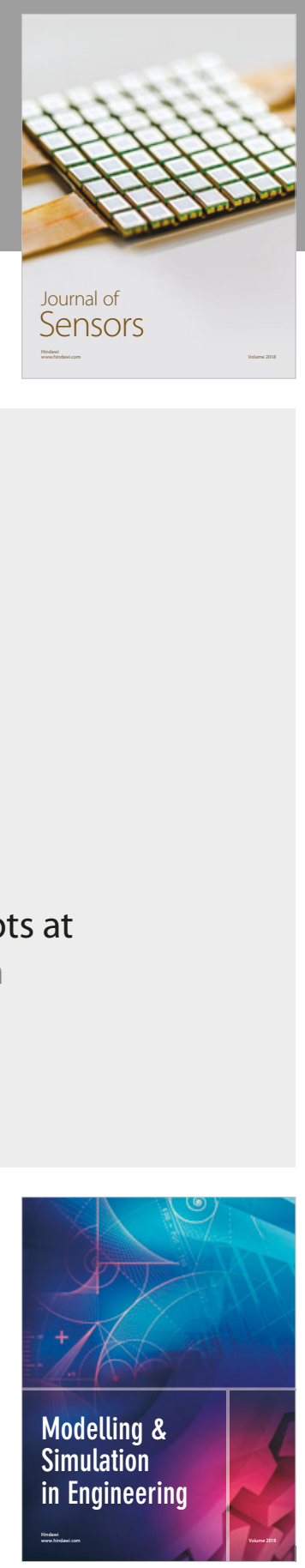

\section{Advances \\ Multimedia}
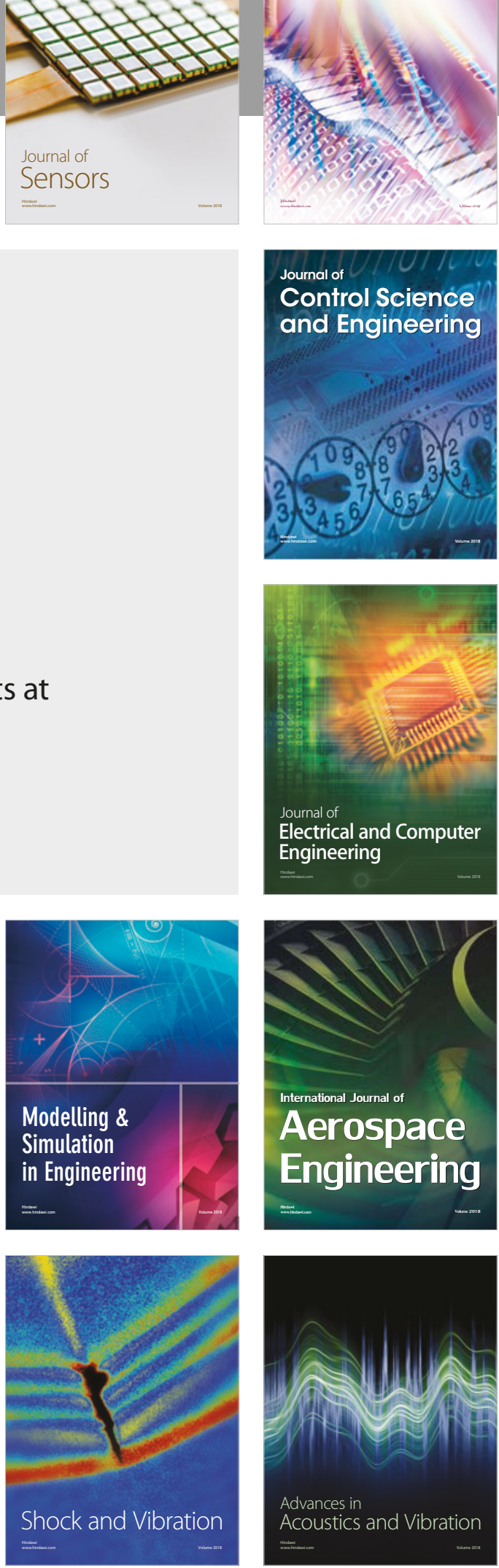